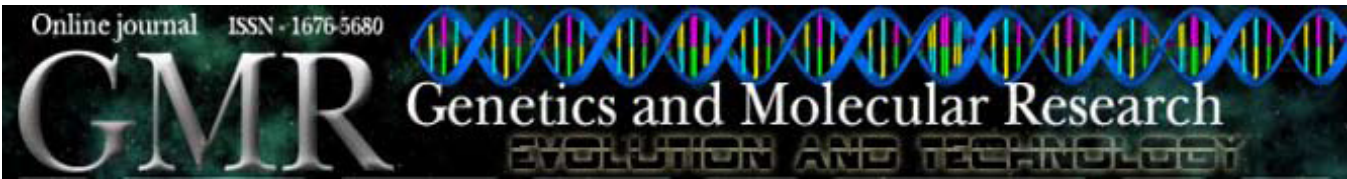

\title{
Anthranilate synthase subunit organization in Chromobacterium violaceum
}

\author{
C.A. Carminatti ${ }^{1}$, I.L. Oliveira ${ }^{1}$, D.O.S. Recouvreux ${ }^{1}$, \\ R.V. Antônio ${ }^{2}$ and L.M. Porto ${ }^{1}$ \\ ${ }^{1}$ Grupo de Engenharia Genômica, Departamento de Engenharia Química \\ e Engenharia de Alimentos, Universidade Federal de Santa Catarina, \\ Florianópolis, SC, Brasil \\ ${ }^{2}$ Grupo de Engenharia Genômica, Departamento de Bioquímica, \\ Universidade Federal de Santa Catarina, Florianópolis, SC, Brasil
}

Corresponding author: C.A. Carminatti

E-mail: claudimir@intelab.ufsc.br

Genet. Mol. Res. 7 (3): 830-838 (2008)

Received June 2, 2008

Accepted August 11, 2008

Published September 16, 2008

\begin{abstract}
Tryptophan is an aromatic amino acid used for protein synthesis and cellular growth. Chromobacterium violaceum ATCC 12472 uses two tryptophan molecules to synthesize violacein, a secondary metabolite of pharmacological interest. The genome analysis of this bacterium revealed that the genes $\operatorname{trp} A-F$ and $p a b A-B$ encode the enzymes of the tryptophan pathway in which the first reaction is the conversion of chorismate to anthranilate by anthranilate synthase (AS), an enzyme complex. In the present study, the organization and structure of AS protein subunits from $C$. violaceum were analyzed using bioinformatics tools available on the Web. We showed by calculating molecular masses that AS in $C$. violaceum is composed of $\alpha(\operatorname{TrpE})$ and $\beta(\mathrm{PabA})$ subunits. This is in agreement with values determined experimentally. Catalytic and regulatory sites of the AS subunits were identified. The TrpE and PabA subunits contribute to the catalytic site while the TrpE subunit is involved in the allosteric site. Protein models for the TrpE and PabA subunits were built by restraint-based homology modeling using AS enzyme, chains A and B, from Salmonella typhimurium (PDB ID 1I1Q).
\end{abstract}

Key words: Tryptophan; Anthranilate synthase; Homology modeling; Chromobacterium violaceum 


\section{INTRODUCTION}

Tryptophan is an aromatic amino acid naturally synthesized by some microorganisms, and it is used for protein synthesis and cellular growth. In most bacteria, tryptophan biosynthesis from chorismate involves five enzymatic reactions catalyzed by five enzymes (Dosselaere and Vanderleyden, 2001; Xiu et al., 2002). Figure 1 shows a general scheme of the tryptophan biosynthesis pathway. In prokaryotes, these proteins are encoded by seven genes, namely $\operatorname{trp} A-G$ (Romero et al., 1995), and they are either expressed individually or as fused units (Table 1). An individual gene may express two or more catalytic domains (Xie et al., 2003). In some bacteria, genes that encode the enzymes for tryptophan biosynthesis are grouped and form the trp operon.

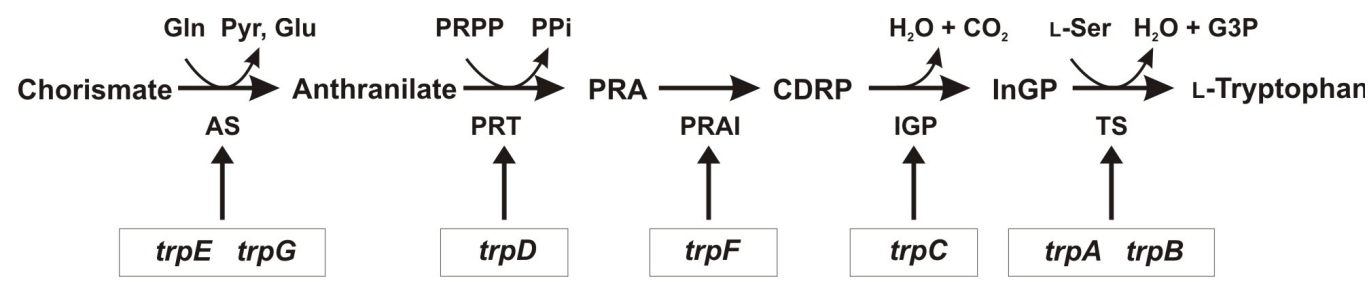

Figure 1. Typical tryptophan biosynthesis pathway in bacteria. AS $=$ anthranilate synthase; PRT $=$ anthranilatephosphoribosyl transferase; PRAI = phosphoribosyl-anthranilate isomerase; IGP = indole glycerol phosphate synthase; TS = tryptophan synthase; PRA = 5-phosphoribosyl anthranilate; CDRP =1-(o-carboxyphenylamino)-1-deoxyribulose5-phosphate; InGP = indole-3-glycerol phosphate; Gln = glutamine; Pyr = pyruvate; Glu = glutamate; PRPP = 5-phosphoribosyl-1-pyrophosphate; $\mathrm{PPi}=$ pyrophosphate; $\mathrm{L}-\mathrm{Ser}={ }_{\mathrm{L}}$-serine; $\mathrm{G} 3 \mathrm{P}=$ glyceraldehyde 3 -phosphate .

Table 1. Protein domains in tryptophan and folate biosynthesis genes of most bacteria.

\begin{tabular}{lll}
\hline Function & Gene & Protein domain encoded \\
\hline Tryptophan biosynthesis & $\operatorname{trpE}$ & anthranilate synthase, aminase subunit $(\alpha)$ \\
& $\operatorname{trpG}$ & anthranilate synthase, amidotransferase subunit $(\beta)$ \\
& $\operatorname{trpD}$ & anthranilate-phosphoribosyl transferase \\
& $\operatorname{trpF}$ & phosphoribosyl-anthranilate isomerase \\
$\operatorname{trpC}$ & $\operatorname{trp} A$ & tryptophan synthase, $\alpha$ subunit \\
& $\operatorname{trpB}$ & tryptophan synthase, $\beta$ subunit \\
& $\operatorname{trpD}(G)$, & \\
$\operatorname{trp} C(F)$, & fusions of domains listed above \\
$\operatorname{trpE}(G)$, & \\
$\operatorname{trp} C(D)$ & $\operatorname{pabB}$ & 4-amino-4-deoxychorismate synthase, aminase subunit $(\alpha)$ \\
$\operatorname{pabA}$ & 4-aminobenzoate synthase, amidotransferase subunit $(\beta)$ \\
& $\operatorname{pabC}$ & 4-amino-4-deoxychorismate lyase $(\gamma)$ subunit \\
\hline
\end{tabular}

Chromobacterium violaceum (ATCC 12472) is a Gram-negative $\beta$-proteobacterium, commonly found in tropical and subtropical regions. Violacein, the characteristic pigment that gives it its name, has been shown to act as an antibiotic and potential antitumor drug (Duran and Menck, 2001; Andrighetti-Fröhner et al., 2003; Antonio and Creczynski-Pasa, 2004). In 
C. violaceum, tryptophan is a precursor for the biosynthesis of violacein, a secondary metabolite. In its genome, the genes $\operatorname{trp} A-F$ and $p a b A-B$, which encode the tryptophan biosynthesis pathway enzymes have been identified. The pabA-B genes also encode folate pathway enzymes. According to the current annotation, $\operatorname{trp} E$, $p a b A$ and $p a b B$ gene products are anthranilate synthase (AS) subunits, and ORF CV0568 is annotated as a probable AS (Brazilian National Genome Project Consortium, 2003).

The first reaction in the tryptophan pathway is catalyzed by AS, which is responsible for the anthranilate synthesis from chorismate and glutamine, a reaction that is feedbackinhibited by tryptophan (Knochel et al., 1999). In most bacteria, AS is an oligomeric enzyme composed of two different subunits - $\alpha$ and $\beta$, which can form an $\alpha \beta$ dimer or an $\alpha_{2} \beta_{2}$ tetramer. The $\alpha$ subunit is encoded by the $\operatorname{trp} E$ gene, while the $\beta$ subunit can be encoded by either $\operatorname{tr} p G$, trpD and/or pabA genes (Dosselaere and Vanderleyden, 2001).

In the present study, computational investigations were performed in order to analyze the structure and organization of AS encoding genes in C. violaceum by the use of bioinformatics tools available on the Web. Amino acid sequences of the TrpE, PabA, PabB, and ORF CV0568 proteins were compared, and TrpE and PabA structures were built using homology molecular modeling.

\section{MATERIAL AND METHODS}

Tryptophan pathway biosynthesis genes of $C$. violaceum, Escherichia coli, Pseudomonas aeruginosa, Pseudomonas putida, Bacillus subtilis, and Salmonella typhimurium were used in this study. The sequences which encode the enzymes of tryptophan synthesis were analyzed by bioinformatics tools available on the Web, including GenBank resources (www.ncbi.nih.gov), Brazilian Virtual Genome Institute of Genomic Research (BRGene) (www.brgene.lncc.br), and the Pathway-Genome Databases EcoCyc and CvioCyc, both available at http://biocyc.org. CvioCyc was built from Pathway-Tools, a software suite developed by SRI International (Karp et al., 2002). The amino acid sequences of microorganisms were submitted to the ClustalW program for multiple alignment (Thompson et al., 1994), using the bioinformatics interface Biological Sequence Alignment Editor (BioEdit) (Tippmann, 2004) (www.mbio.ncsu.edu/BioEdit/BioEdit.html).

In order to model the proteins of interest, the corresponding amino acid sequences were submitted to the Swiss-Model database (Guex and Peitsch, 1997) to obtain a model protein with a high degree of similarity ( $>25 \%$ ) deposited in the Protein Data Bank (PDB) (Berman et al., 2000). The alignment of the target proteins against the template sequences was used as input to the Modeller 8v2 program (Sali and Blundell, 1993), together with the atomic coordinates of the latter. The file with the spatial coordinates of the best model generated by ModeLLER was visualized by SPDBViewer (Guex and Peitsch, 1997), as a protein three-dimensional model. Model validation was done by VERIFY-3D, a structure evaluation server (Luthy et al., 1992) and Procheck (Laskowski et al., 1993), a computational tool that analyzes the stereochemical quality of the protein through the linking lengths, torsional angles of the main chain, and Ramachandran diagrams.

\section{RESULTS AND DISCUSSION}

Four amino acid sequences have been annotated in the $C$. violaceum genome as belonging to the AS enzyme subunits: TrpE - AS ( $\alpha$ subunit), PabA - AS ( $\beta$ subunit), PabB - AS, and ORF CV0568 - a probable AS (Brazilian National Genome Project Consortium, 2003). 
The conserved sequences LLESX ${ }_{10} \mathrm{~S}$ and NPSPYM in the TrpE subunit identify the tryptophan binding site, which is associated with regulatory functions such as inhibiting the AS activity (Dosselaere and Vanderleyden, 2001). The comparison of these conserved sequences with AS amino acid sequences of other bacteria demonstrated that the TrpE is the only annotated sequence in AS subunits from C. violaceum that possesses those consensuses (Figure 2). Therefore, based on the alignment described in Figure 2, and due to the existence of the inhibitory tryptophan binding site, we suggest that TrpE is a $C$. violaceum $\mathrm{AS}$ subunit.

\begin{tabular}{|c|c|c|c|c|}
\hline TrpE CV & ........ AQPYSY & ELESVVGGERHG RYS & IG.....RALRNI NPSPYM & FFYHF........ \\
\hline TrpE & ...... ADAPNSY & LLESVQGGEK WGRYS & IG.....RALRCENPTPYM & IFFNE............ \\
\hline TrpE & ...... GDRPATI & LLESADIDSK DDLKS & LLL.....YVLKKSNPSPYM & 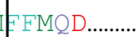 \\
\hline TrpE BS & ...... DREITY & LLESKDDTSTW SRYS & IG......RVLRIVNPSPYM & 1 YMKLL.......... \\
\hline
\end{tabular}

Figure 2. Alignment showing the inhibitory tryptophan binding site (LLESX ${ }_{10} \mathrm{~S}$ and NPSPYM conserved sequences). $C v=$ Chromobacterium violaceum; $P a=$ Pseudomonas aeruginosa $; E c=$ Escherichia coli; $B s=$ Bacillus subtilis .

Wegman and Crawford (1968) have shown by experimental techniques that the molecular mass of AS from C. violaceum must be between 84 to $95 \mathrm{kDa}$. Taking this range into account, the AS structure should have a molecular mass close to the sum of the molecular mass of TrpE and one of the other subunits. The molecular mass of each subunit was estimated from the protein primary sequence, giving 54,022 Da for TrpE, 47,930 Da for CV0568, 21,048 Da for PabA, and 47,877 Da for PabB. Hence, the AS subunits should be TrpE and PabA, since the sum of their molecular masses $(75,070 \mathrm{Da})$ is close to the experimental values found for the AS protein complex. To confirm this hypothesis, the AS catalytic domains were compared with AS domains from other bacteria.

In the TrpE subunit from S. typhimurium, a histidine residue that is essential for catalytic activity was found at position 398 (Tso and Zalkin, 1981). The TrpE alignment from $P$. aeruginosa, B. subtilis and $S$. typhimurium with TrpE from C. violaceum demonstrated that there is a histidine residue at position 367 (Figure 3). The same alignment was carried out with ORF CV0568. Figure 4 shows His in this protein at position 333. A query with the CV0568 sequence to the Pfam database (www.sanger.ac.uk/Software/ Pfam) showed that the protein encoded by this ORF belongs to the chorismate binding family. Therefore, TrpE and CV0568 belong to an enzyme family that has chorismate as a common substrate.

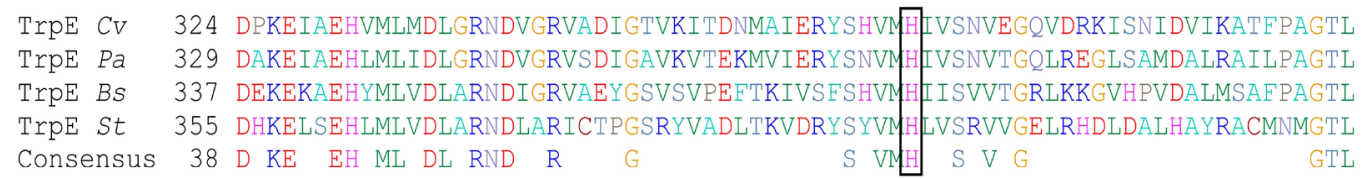

Figure 3. Alignment showing the conserved histidine at position 367 in the TrpE from Chromobacterium violaceum. $C v=C$. violaceum $; \mathrm{Pa}=$ Pseudomonas aeruginosa $B s=$ Bacillus subtilis; St $=$ Salmonella typhimurium . 


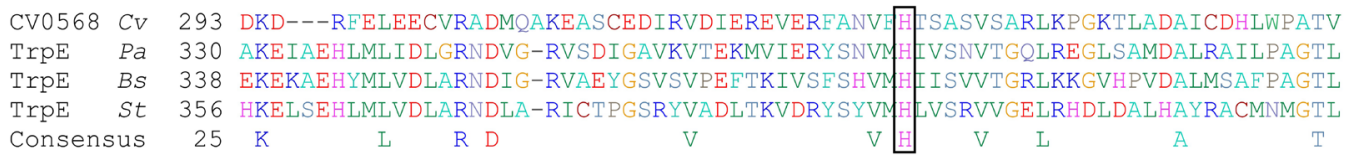

Figure 4. Alignment showing the conserved histidine at position 333 in ORF CV0568 from Chromobacterium violaceum. $C v=C$. violaceum; $P a=$ Pseudomonas aeruginosa; $B s=$ Bacillus subtilis; $S t=$ Salmonella typhimurium.

The AS $\beta$ subunit belongs to the glutamine amidotransferase family (GATases), where three amino acids are highly conserved - cysteine $(\mathrm{C})$, histidine $(\mathrm{H})$ and glutamate $(\mathrm{E})$ - forming a catalytic triad (Massiere and Badet-Denisot, 1998). In P. putida, a cysteine residue was identified at position 79 (Cys79), while in E. coli the histidine residue was found at position 170 (His 170). The alignment of E. coli (TrpD), P. putida (TrpG), B. subtilis (PabA), and PabA from C. violaceum revealed the existence of the conserved residues Cys79, His 168 and Glu170 (Figure 5). Thus, the PabA subunit also belongs to the GATase family. Those three residues were not found to be conserved next to the corresponding positions in ORF CV0568 (data not shown). Therefore, we conclude that: a) AS from C. violaceum is probably composed of an $\alpha$ subunit (TrpE) and a $\beta$ subunit (PabA); b) the TrpE and PabA subunits bear a catalytic function, and c) the TrpE subunit has an allosteric site (tryptophan binding site).

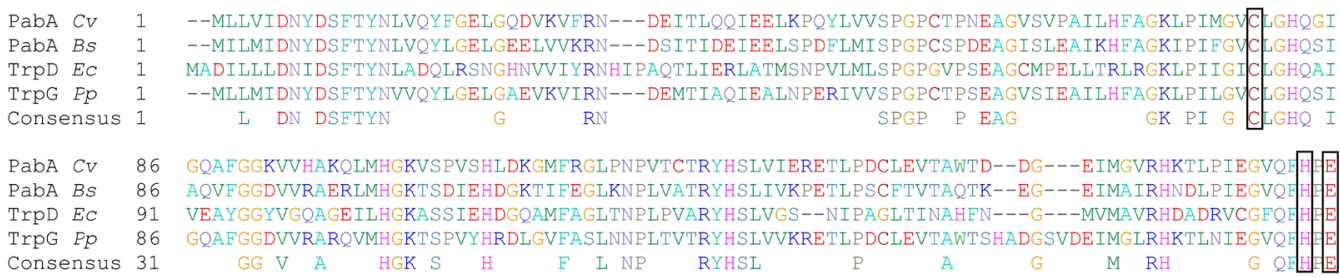

Figure 5. Alignment showing conserved cysteine $(\mathrm{C})$, histidine $(\mathrm{H})$ and glutamate $(\mathrm{E})$ residues in PabA, TrpD and TrpG sequences. $C v=$ Chromobacterium violaceum; $B s=$ Bacillus subtilis; Ec $=$ Escherichia coli; $P p=$ Pseudomonas putida.

To obtain a three-dimensional model of AS subunits from C. violaceum, TrpE and PabA protein sequences were submitted to the Swiss-Model database to search for a protein template with high similarity in the PDB. The AS template from S. typhimurium (Morollo and Eck, 2001) (PDB ID 1I1Q), chains A and B, revealed 39 and 41\% identity when compared to TrpE and PabA from C. violaceum, respectively.

ClustalW aligned sequences were submitted to ModelLer, and the results were visualized by using the SPDBViewer software. Figure 6 shows protein template 1I1QA and the modeled protein for the TrpE subunit. The generated model and template were superposed using SPDBViewer, and the amino acid residue histidine, conserved at position His398 for $S$. typhimurium and His367 for $C$. violaceum was found in both proteins at the same spatial position (See Figure 6). The conserved sequences LLESX ${ }_{10} \mathrm{~S}$ and NPSPYM were found in TrpE from C. violaceum at positions Leu45-Leu46-Glu47-Ser48-X ${ }_{10}$-Ser59 and Asn265-Pro266Ser267-Pro268-Tyr269-Met270, confirming the existence of the tryptophan binding site (data not shown). 

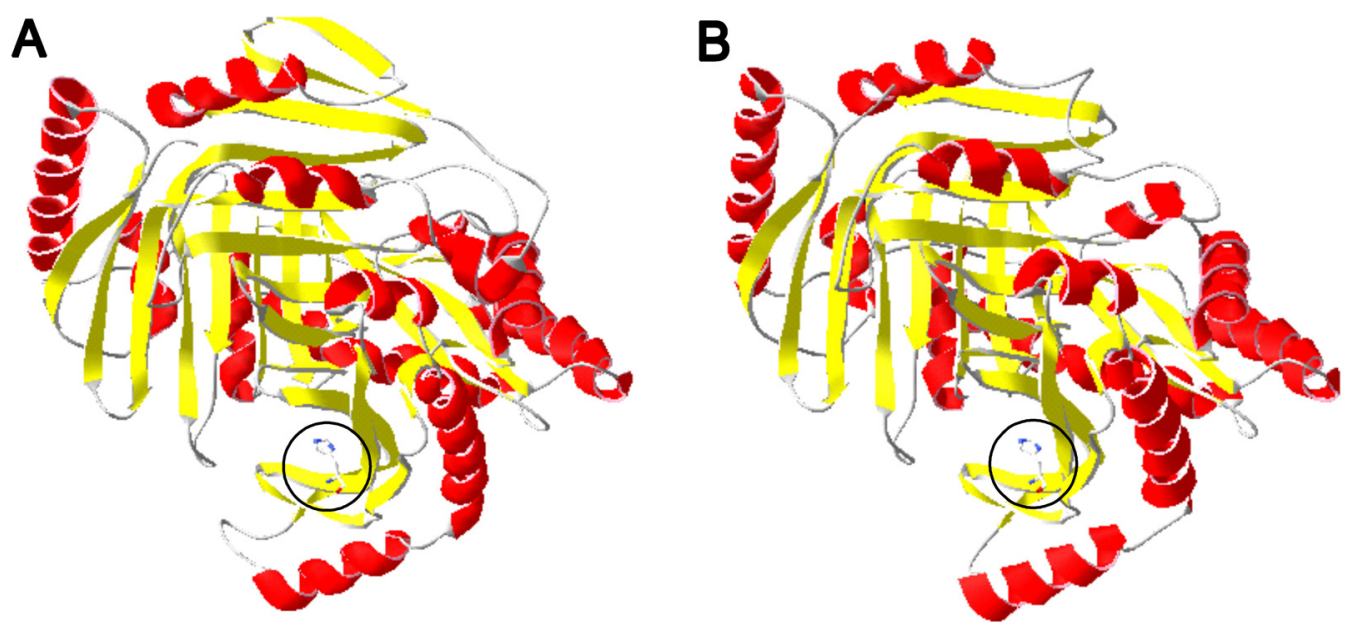

Figure 6. A. Structure of the template - TrpE protein (1I1QA) from Salmonella typhimurium determined by X-ray crystallography (Morollo and Eck, 2001). B. Homology model of TrpE subunit from Chromobacterium violaceum. Position of histidine in both proteins is shown inside the circles.

The TrpG protein (1I1QB) from S. typhimurium was used as template for modeling the $C$. violaceum PabA subunit. Figure 7 shows the template and modeled proteins. Amino acid residues found in S. typhimurium TrpG protein at positions Cys83, His169 and Glu171 were found in C. violaceum PabA subunit at positions Cys79, His168 and Glu170. These residues correlate perfectly upon superposition, and they form the catalytic site where the conversion of chorismate to anthranilate occurs (See Figure 7).
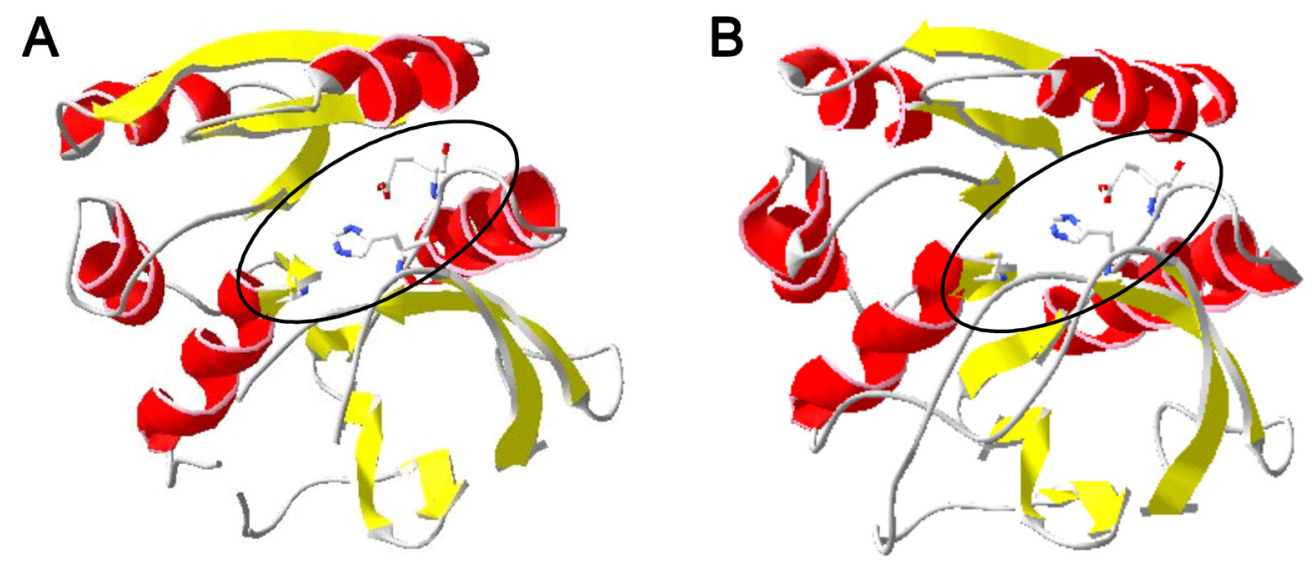

Figure 7. A. Structure of the template - TrpG protein (1I1QB) from Salmonella typhimurium determined by X-ray crystallography (Morollo and Eck, 2001). B. Homology model of PabA subunit from Chromobacterium violaceum. Positions of cysteine, histidine and glutamate in both proteins are shown inside the ovals. 
The AS TrpE and PabA model structure from $C$. violaceum was validated by VERIFY3D (Figure 8). The compatibility scores above zero in the VERIFY-3D graph correspond to acceptable side chain environments. We can see that most of the 3D-1D average scores are above zero, computational evidence that the $C$. violaceum TrpE and PabA subunits built from homology modeling are acceptable.
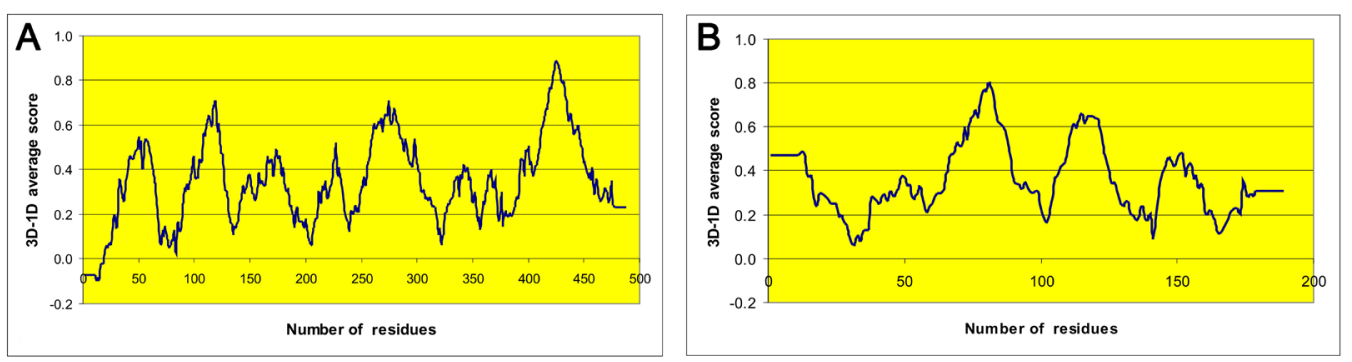

Figure 8. 3D-1D average score profiles used to verify results of anthranilate synthase subunit models from Chromobacterium violaceum. Residues with positive compatibility scores are reasonably folded. A. TrpE. B. PabA.

The subunit models for TrpE and PabA were also validated using Ramachandran diagrams. A high-quality modeled protein should have more than $90 \%$ of its residues located in the most favorable region (Ramachandran et al., 1963). For the TrpE subunit, $98.5 \%$ of amino acid residues are in favorable energetic regions, while for PabA they represent $96.8 \%$ of the residues. Figure 9 presents a Ramachandran plot for AS enzyme, TrpE and PabA subunits, for C. violaceum.
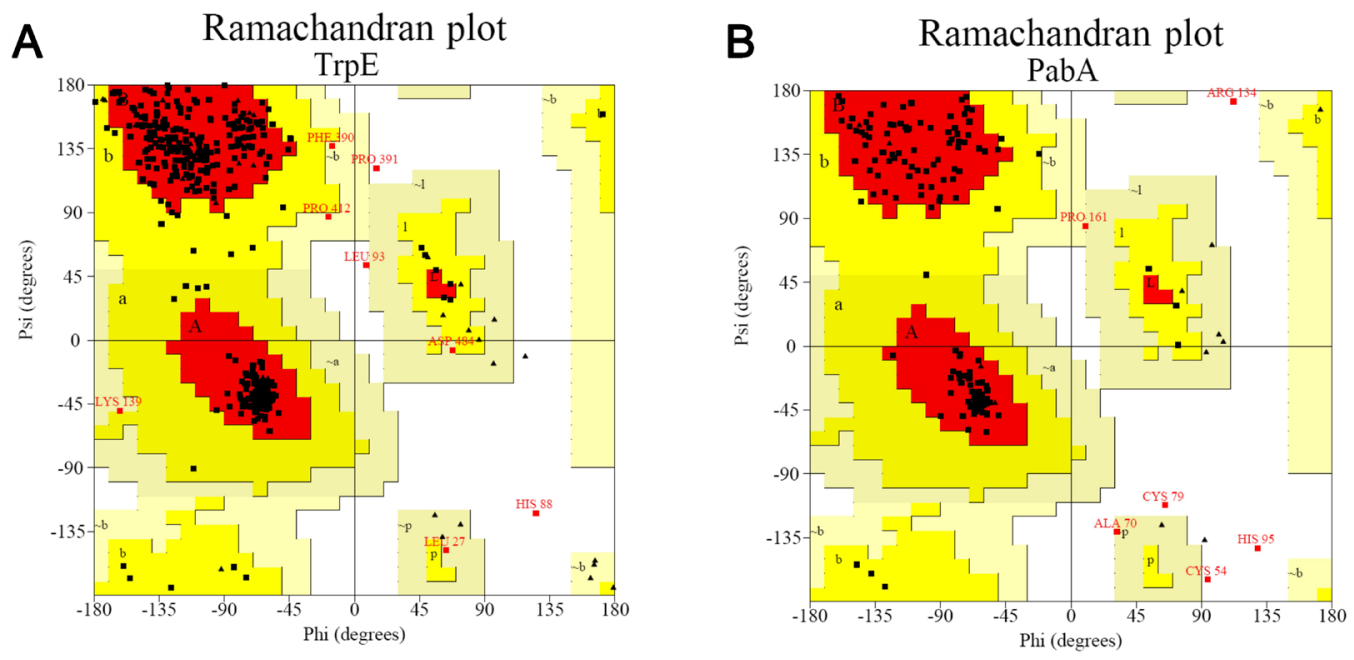

Figure 9. Ramachandran diagrams for anthranilate synthase subunits TrpE (A) and PabA (B). Amino acids (98.5 and $96.8 \%$ ) are in favorable regions for TrpE and PabA proteins from Chromobacterium violaceum, respectively. 


\section{CONCLUDING REMARKS}

The study of the tryptophan biosynthesis pathway in C. violaceum is important since tryptophan is the main precursor of violacein, the characteristic pigment of this bacterium, which possesses potential antibiotic, antitumor and antiparasitic applications.

Using computational tools, the analysis of TrpE, PabA, PabB, and ORF CV0568 protein sequences revealed that the AS from $C$. violaceum is composed of an $\alpha$ subunit encoded by the $\operatorname{trp} E$ gene and a $\beta$ subunit encoded by the $p a b A$ gene. The sum of the molecular mass for those subunits is close to the experimental values, as determined by Wegman and Crawford (1968). TrpE and PabA subunits bear catalytic sites and the TrpE subunit contains the allosteric site. $p a b B$ gene and ORF CV0568, currently annotated as being an AS subunit and a probable AS, respectively, are probably not AS enzyme subunits. Instead, our results suggest that ORF CV0568 is a chorismate binding enzyme family member.

The TrpE and PabA subunits from C. violaceum obtained by homology modeling using AS (TrpE and TrpG subunits) as templates from S. typhimurium showed high stereochemical quality. Most of the amino acid residues of AS from C. violaceum $(98.5 \%$ for $\operatorname{TrpE}$ and $96.8 \%$ for PabA) are in favorable regions. Catalytic and allosteric sites were found in the modeled structures, suggesting that the AS enzyme from C. violaceum is probably composed of two subunits, TrpE and PabA.

\section{ACKNOWLEDGMENTS}

The authors thank the reviewers for their very helpful comments and suggestions. Research partially supported by Conselho Nacional de Desenvolvimento Científico e Tecnológico $(\mathrm{CNPq})$.

\section{REFERENCES}

Andrighetti-Fröhner CV, Antonio RV, Pasa TC, Barardi CRM, et al. (2003). Cytotoxicity and potential antiviral evaluation of violacein produced by Chromobacterium violaceum. Mem. Inst. Oswaldo Cruz 98: 843-848.

Antonio RV and Creczynski-Pasa TB (2004). Genetic analysis of violacein biosynthesis by Chromobacterium violaceum. Genet. Mol. Res. 3: 85-91.

Berman HM, Westbrook J, Feng Z, Gilliland G, et al. (2000). The protein Data Bank. Nucleic Acids Res. 28: 235-242.

Brazilian National Genome Project Consortium (2003). The complete genome sequence of Chromobacterium violaceum reveals remarkable and exploitable bacterial adaptability. Proc. Natl. Acad. Sci. U. S. A. 100: 11660-11665.

Dosselaere F and Vanderleyden J (2001). A metabolic node in action: chorismate-utilizing enzymes in microorganisms. Crit. Rev. Microbiol. 27: 75-131.

Duran N and Menck CF (2001). Chromobacterium violaceum: a review of pharmacological and industrial perspectives. Crit. Rev. Microbiol. 27: 201-222.

Guex N and Peitsch MC (1997). SWISS-MODEL and the Swiss-PdbViewer: an environment for comparative protein modeling. Electrophoresis 18: 2714-2723.

Karp PD, Paley S and Romero P (2002). The Pathway Tools software. Bioinformatics 18 (Suppl 1): S225-S232.

Knochel T, Ivens A, Hester G, Gonzalez A, et al. (1999). The crystal structure of anthranilate synthase from Sulfolobus solfataricus: functional implications. Proc. Natl. Acad. Sci. U. S. A. 96: 9479-9484.

Laskowski RA, MacArthur MW, Moss DS and Thornton JM (1993). PROCHECK: a program to check the stereochemical quality of protein structures. J. Appl. Crystallogr. 26: 283-291.

Luthy R, Bowie JU and Eisenberg D (1992). Assessment of protein models with three-dimensional profiles. Nature 356: $83-85$. 
Massiere F and Badet-Denisot MA (1998). The mechanism of glutamine-dependent amidotransferases. Cell Mol. Life Sci. 54: 205-222.

Morollo AA and Eck MJ (2001). Structure of the cooperative allosteric anthranilate synthase from Salmonella typhimurium. Nat. Struct. Biol. 8: 243-247.

Ramachandran GN, Ramakrishnan C and Sasisekharan V (1963). Stereochemistry of polypeptide chain configurations. $J$. Mol. Biol. 7: 95-99.

Romero RM, Roberts MF and Phillipson JD (1995). Anthranilate synthase in microorganisms and plants. Phytochemistry 39: $263-276$.

Sali A and Blundell TL (1993). Comparative protein modelling by satisfaction of spatial restraints. J. Mol. Biol. 234: 779-815.

Thompson JD, Higgins DG and Gibson TJ (1994). Clustal W: improving the sensitivity of progressive multiple sequence alignment through sequence weighting, position-specific gap penalties and weight matrix choice. Nucleic Acids Res. 22: $4673-4680$.

Tippmann HF (2004). Analysis for free: comparing programs for sequence analysis. Brief. Bioinform. 5: 82-87.

Tso JY and Zalkin H (1981). Chemical modifications of Serratia marcescens anthranilate synthase component I. J. Biol. Chem. 256: 9901-9908.

Wegman J and Crawford IP (1968). Tryptophan synthetic pathway and its regulation in Chromobacterium violaceum. J. Bacteriol. 95: 2325-2335.

Xie G, Keyhani NO, Bonner CA and Jensen RA (2003). Ancient origin of the tryptophan operon and the dynamics of evolutionary change. Microbiol. Mol. Biol. Rev. 67: 303-342.

Xiu ZL, Chang ZY and Zeng AP (2002). Nonlinear dynamics of regulation of bacterial trp operon: model analysis of integrated effects of repression, feedback inhibition, and attenuation. Biotechnol. Prog. 18: 686-693. 